04

\title{
Аппаратно-алгоритмическое обеспечение корреляционного детектирования в голографических датчиках волнового фронта
}

\author{
(C) П.А. Ручка ${ }^{1}$, Н.М. Вереникина ${ }^{1}$, И.В. Гриценко ${ }^{1}$, Е.Ю. Злоказов ${ }^{1,2}$, М.С. Ковалев ${ }^{1}$, Г.К. Красин ${ }^{1}$, \\ С.Б. Одиноков ${ }^{1,{ }^{\top}}$, Н.Г. Сцепуро ${ }^{1}$ \\ ${ }^{1}$ МГТУ им. Н.Э. Баумана, \\ 105005 Москва, Россия \\ ${ }^{2}$ Национальный исследовательский ядерный университет „МИФИ“, \\ 115409 Москва, Россия \\ I e-mail: odinokov@bmstu.ru
}

Поступила в редакцию 31.05.2019 г.

В окончательной редакции 31.05.2019 г.

Принята к публикации 04.06.2019 г.

Описан алгоритм, который позволяет в автоматическом режиме вычислять аберрации в предложенной авторами ранее схеме голографического датчика волнового фронта, позволяющей измерять аберрации на основе итерационного вывода голограмм на фазовый пространственный модулятор света (ПМС). Приведена практическая реализация данного алгоритма на основе обратной связи камеры и ПМС. С использованием предлагаемого алгоритма измерена дефокусировка с точностью $\lambda / 160$.

Ключевые слова: компьютерная голография, голограмма Фурье, измерение волнового фронта, датчик волнового фронта, корреляционное детектирование.

DOI: $10.21883 /$ OS.2019.10.48358.172-19

\section{Введение}

Прецизионные измерения искажений волнового фронта играют большую роль во многих областях фотоники: контроль волнового фронта лазерного излучения, оптический контроль качества поверхностей, измерение аберраций человеческого глаза, оптическая микроскопия, атмосферная оптика, оптические линии связи и др. [1-5].

В последние годы большой прогресс был достигнут в области создания, исследования и применения еще одного типа оптических приборов, а именно датчиков волнового фронта (ДВФ). Этот термин применяется к группе устройств, обеспечивающих измерение отклонения волнового фронта оптического излучения от плоскости или сферы. В [6] была показана схема голографического датчика волнового фронта (ГДВФ), принцип работы которого заключался в анализе сигнала, сформированного при подсвете голографического фильтра пучком света. Этот фильтр выделял амплитуду полинома Цернике, и, таким образом, вычислялась аберрация волнового фронта, которая соответствовала данному полиному. Такой подход положил начало развитию ГДВФ в приложениях атмосферной адаптивной оптики, так как авторами была показана возможность измерения быстро осциллирующих аберраций волнового фронта. Впоследствии были предложены и другие похожие схемы ГДВФ [7-15], которые позволили расширить динамический диапазон, уменьшить погрешность, увеличить скорость измерений и снизить влияние кросс-модуляционных шумов. Однако так и не удалось преодолеть ключевые ограничения таких схем $[16,17]$.

В работах $[18,19]$ была предложена схема ГДВФ, который измеряет искажения волнового фронта на основе компьютерно-синтезированных голограмм Фурье (КСГ). Детектирование фазовых искажений, присутствующих в лазерном пучке, осуществляется с помощью специальной голограммы Фурье, которая представляет собой результат интерференции двух волн. На рис. 1 представлена схема синтезирования такой голограммы. Первая волна, предметная, формируется с использованием точечного источника $S$, смещенного относительно оптической оси на величину $\Delta$. Сферическая волна, исходящая от источника, подвергается фурье-преобразованию и падает на фоточувствительную пластинку $h$.

Вторая волна, опорная, $r$ представляет собой когерентный пучок единичной амплитуды с фазовым множителем $f_{r e f}(x, y)$, который представляет собой функцию, описывающую аберрации, внесенные в опорную волну.

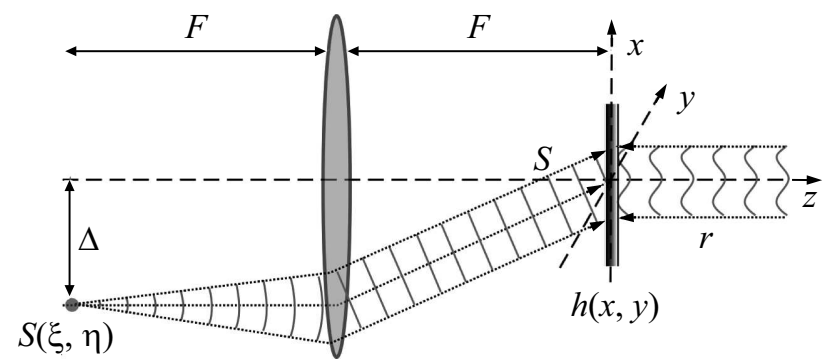

Рис. 1. Эквивалентная схема записи амплитудной голограммы Фурье. 
В настоящей работе в качестве модели фазовых искажений использовались полиномы Цернике. Также для описания аберраций могут быть использованы функции Карунена-Лоэва, полиномы Чебышева или другие ортогональные базисы [20].

Этот ГДВФ в перспективе может использоваться в задачах, где требуется проводить прецизионные измерения фазовых искажений волнового фронта. Принцип построения такого голографического датчика заключался в формировании корреляционного отклика в плоскости приемника излучения, когда модель закодированного в голограмму волнового фронта соответствовала функции волнового фронта измеряемого луча. Аберрации определялись посредством динамической корректировки весовых коэффициентов ортонормированного базиса модели волнового фронта в голограмме для получения максимальной амплитуды отклика. Однако такая аппроксимация посредством перебора значений занимает продолжительное время и может привести к возникновению погрешностей. Таким образом, необходимо автоматизировать этот процесс, а также выработать оптимальную целевую функцию оптимизации и алгоритма аппроксимации коэффициентов базиса.

В настоящей работе предлагается аппаратно-алгоритмическое обеспечение голографического датчика волнового фронта с корреляционным детектированием фазовых искажений на основе компьютерно-синтезированных голограмм Фурье. Представлена модификация предложенной ранее схемы, которая позволяет измерять фазовые искажения волнового фронта в автоматическом режиме на основе обратной связи камеры и пространственного модулятора света, иными словами, - осуществить временное мультиплексирование голограмм. Рассмотрены способ задания целевой функции для корреляционного детектирования и алгоритм автоматического измерения волновых аберраций на основе регистрируемого корреляционного отклика.

\section{Детектирование фазовых искажений на основе корреляционного отклика}

При восстановлении голограммы Фурье лазерным пучком с фазовыми искажениями излучение дифрагирует на несколько порядков на камере, которая стоит в задней фокальной плоскости фурье-объектива (рис. 2). По интенсивности и размеру данных порядков дифракции можно судить о наличии и величине аберрации, содержащейся в падающем пучке. Однако для прецизионного измерения предлагается работать не с аналоговыми голограммами Фурье, которые могут вносить свои погрешности и нелинейности при записи, а рассчитывать компьютерно-синтезированные голограммы Фурье и выводить их на пространственном модуляторе света для существенного уменьшения погрешности определения фазовых искажений.

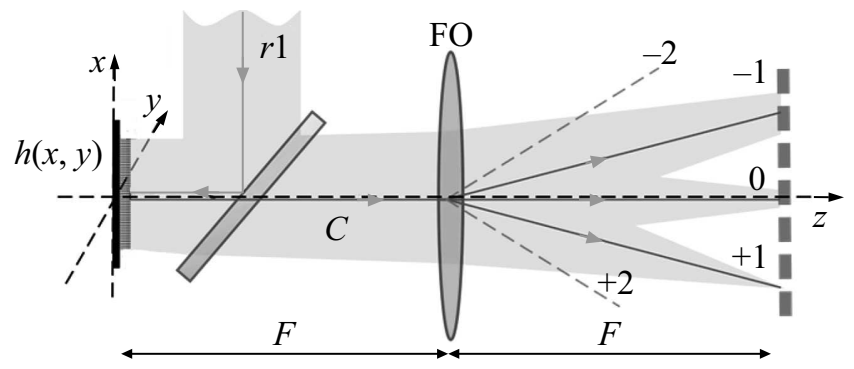

Рис. 2. Оптическая схема восстановления голограммы Фурье при падении на нее лазерного пучка с фазовыми искажениями.

Функция пропускания КСГ может быть рассчитана как квадрат суммы комплексных амплитуд предметной и опорной волн. Запишем нормированную функцию пропускания голограммы, которая отображается на фазовом ПМС:

$$
h_{\mathrm{ph}}(x, y) \sim \exp \left\{i \pi \cos \left[\frac{2 \pi}{\lambda}\left(\frac{\Delta}{F} x-f_{\text {ref }}(x, y)\right)\right]\right\},
$$

где $F-$ фокусное расстояние фурье-объектива, $\lambda-$ длина волны.

Согласно формуле Якоби-Ангера [21], можно преобразовать указанное выше выражение как сумму взвешенных функций Бесселя 1-го рода $n$-го порядка. Тогда при падении измеряемого лазерного пучка $t(x, y)=\exp \left[i \frac{2 \pi}{\lambda} f(x, y)\right]$ с неизвестной аберрацией $f(x, y)$ на фазовый ПМС, на который выводится голограмма, получится следующее распределение:

$$
\begin{aligned}
c(x, y) & =t(x, y) h_{\mathrm{ph}}(x, y)=\sum_{n \in Z} i^{n} J_{n}(\pi) \\
& \times \exp \left[i \frac{2 \pi}{\lambda}\left(\frac{n \Delta}{F} x\right)\right] r_{n}^{*}(x, y) t(x, y),
\end{aligned}
$$

где $r_{n}^{*}(x, y)=\exp \left[i \frac{2 \pi}{\lambda} n f_{r e f}(x, y)\right]-$ опорная волна, использовавшаяся для расчета КСГ, $J_{n}(\pi)$ - взвешенные функции Бесселя 1-го рода.

Пространственный модулятор света установлен в передней фокальной плоскости объектива, где формируется световое поле, комплексная амплитуда которого описывается фурье-преобразованием комплексной амплитуды падающего на объектив поля. В задней фокальной плоскости фурье-объектива установлена КМОПкамера, которая регистрирует следующее распределение интенсивности излучения:

$$
\begin{aligned}
& |C(\xi, \eta)|^{2} \sim\left|\tilde{\mathscr{F}}^{-1}[c(x, y)]\right|^{2} \\
& \sim\left|\sum_{n \in Z} i^{n} J_{n}(\pi)\left[R_{n}(\xi-n \Delta, \eta)\right] \otimes T(\xi-n \Delta, \eta)\right|^{2},
\end{aligned}
$$

где $\tilde{\mathscr{F}}$ - оператор преобразования Фурье, $T(\xi, \eta)$ и $R_{n}(\xi, \eta)$ - фурье-образы от функций $t(x, y)$ и $r_{n}(x, y)$ соответственно, а $\otimes-$ знак кросс-корреляции. 
Таким образом, при выводе КСГ на ПМС независимо от конкретной амплитуды соответствующих фазовых искажений в аберрационной модели в плоскости регистрации можно будет наблюдать корреляционный отклик в порядках дифракции [22]. Пространственное распределение интенсивности отклика в плоскости регистрации и его положение, т.е. порядок дифракции, в котором наблюдается отклик, будут изменяться в зависимости от соотношения амплитуд волновых аберраций, кодируемых в КСГ, и амплитуд аберраций в пучке, падающем на ПМС.

При совпадении амплитуд искажений в падающем на пространственный модулятор световом пучке и в функции, описывающей аберрации в КСГ, в плоскости регистрирующей матрицы в +1-м порядке дифракции будет наблюдаться острый пик, амплитуда которого будет соответствовать теоретическому пределу для автокорреляции в данной системе. Важно отметить, что поскольку при синтезе голограммы в качестве объектной волны используется точечный источник света, то корреляционный отклик по своему распределению является изображением функции рассеяния точки данной оптической системы. Это обстоятельство обусловливает также то, что при несовпадении аберраций в пучке и в голограмме, картина в +1-м порядке соответствует виду функции рассеяния точки для соответствующих фазовых искажений.

Таким образом, при рассмотрении картины в +1 -м порядке можно корректировать КСГ на ПМС для получения идеального корреляционного отклика.

\section{Алгоритм детектирования. Метод вычисления аберраций}

Идея автоматического детектирования и измерения искажений волнового фронта с использованием методов компьютерной голографии заключается в создании обратной связи между приемником излучения, регистрирующим корреляционный отклик, и отображающим КСГ фазовым пространственным модулятором света. Обратная связь осуществляется через подключение этих устройств к ПК. Предлагаемый алгоритм на основе измерения амплитуды корреляционного отклика корректирует значение исследуемой аберрации в КСГ, осуществляя ее перерасчет в автоматическом режиме, и снова оценивает максимальную амплитуду отклика. Задача измерения аберраций считается решенной, когда с заданной точностью определены все требуемые для описания волнового фронта коэффициенты полиномов Цернике.

Принимая во внимание вышесказанное, задача вычисления амплитуды каждой аберрации, представленной в виде полинома Цернике, сводится к нахождению максимума функции корреляционного отклика в зависимости от закодированного в КСГ значения амплитуды фазового искажения. Особая сложность задачи заключается в

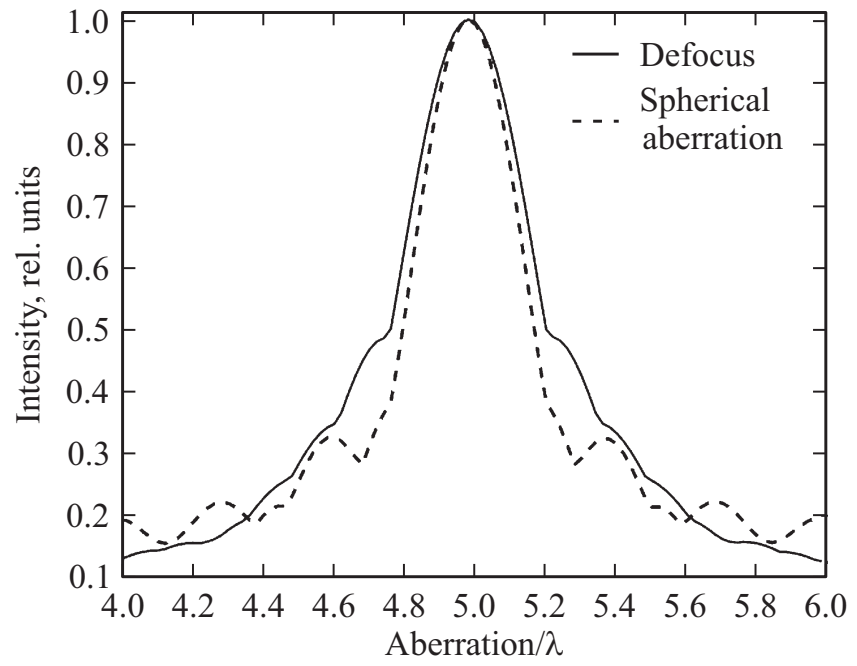

Рис. 3. Теоретический график зависимости амплитуды корреляционного пика от величины аберрации в голограмме: сплошной линией - для пучка с дефокусировкой $5 \lambda$, штрихами для пучка со сферической аберрацией $5 \lambda$.

том, что целевая функция - зависимость отклика от амплитуды искажения в голограмме - не является унимодальной, т. е. имеет несколько экстремумов. Кроме этого, функции для разных аберраций имеют разные ширину, частоту и количество локальных максимумов, что усложняет задачу оптимизации. На рис. 3 представлены теоретические графики зависимости максимума корреляционного отклика от величины аберрации в голограмме для лазерного пучка с дефокусировкой $5 \lambda$ и со сферической аберрацией $5 \lambda$. Можно видеть, что сферическая аберрация имеет более выраженные локальные максимумы, а ширина пика меньше, чем для дефокусировки с аналогичной амплитудой.

Таким образом, целевую функцию можно представить в виде $U_{\max }(\lambda)$, где $U_{\max }-$ величина максимума амплитуды корреляционного пика. Поскольку нам известна целевая функция, а также известны интервал измеряемых значений аберраций и критерий поиска - глобальный максимум, можно утверждать, что обозначенная задача представляет собой классическую задачу математической оптимизации [23]. В настоящее время существует множество алгоритмов оптимизации, применяемых для поиска локальных экстремумов, наиболее известными из которых являются метод золотого сечения, метод деления пополам, метод дихотомии, а также более сложные - например градиентный спуск [24]. С учетом вида целевой функции в нашей задаче при применении тривиальных алгоритмов оптимизации существует высокая вероятность попадания в локальный максимум, особенно при оценке сферической аберрации и комы, в результате чего фазовые искажения будут измерены с большой погрешностью.

Для случаев, когда целевая функция имеет локальные экстремумы, существуют различные методы, позволя- 


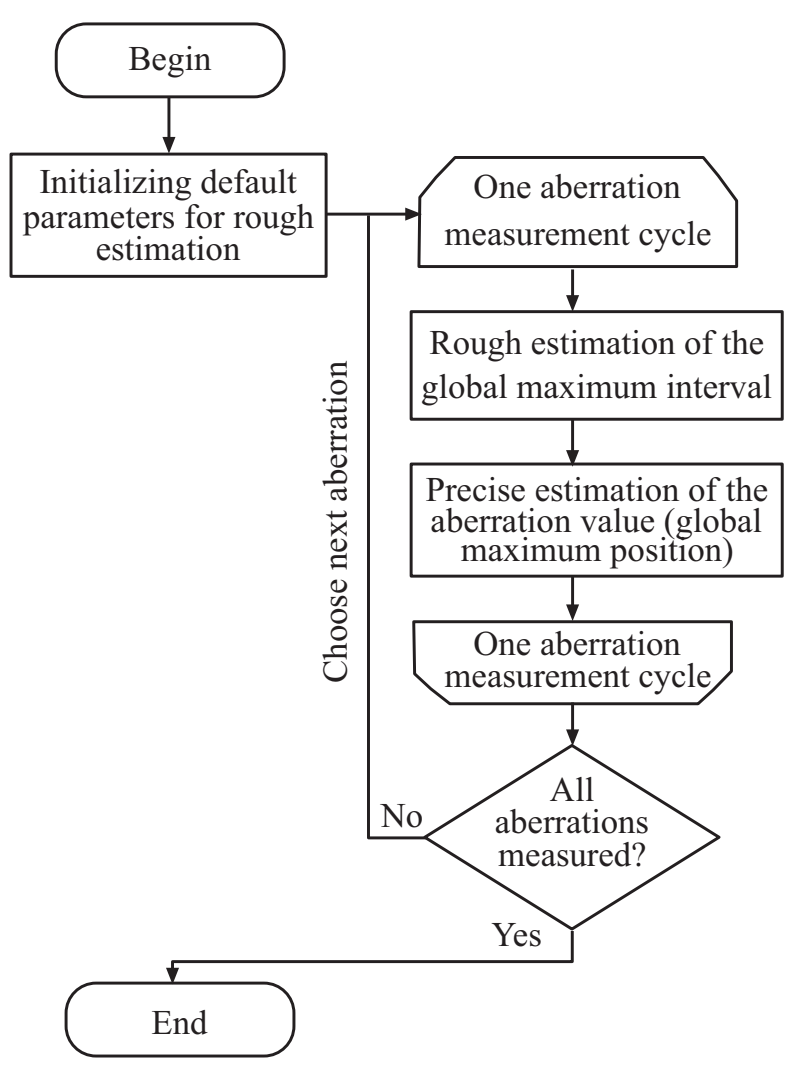

Рис. 4. Блок-схема алгоритма автоматического вычисления аберраций.

ющие определить глобальный максимум, что в нашей задаче соответствует нахождению истинного значения каждой аберрации. Однако многомерная глобальная оптимизация требует не только создания специфических алгоритмов, но и применения крайне мощных вычислительных ресурсов. Поэтому в настоящей работе предлагается более простой метод нахождения значений аберраций.

На рис. 4 представлена блок-схема разработанного для данной задачи алгоритма определения аберраций в исследуемом пучке. Было реализовано специализированное программное обеспечение на языке Python, которое позволило автоматизировать процесс вычислений. Весь процесс вычислений включает в себя два этапа: 1) грубый поиск интервала, содержащего один экстремум - глобальный максимум функции амплитуды корреляционного отклика в зависимости от величины аберрации в голограмме; 2) точное определение положения экстремума.

На первом этапе в интерфейсе программы необходимо задать начальные параметры грубого поиска. Пусть для всех аберраций шаг грубой сетки выбран равным $0.4 \lambda$ в интервале $(0-15) \lambda$. Получим 38 значений каждой аберрации, что соответствует 38 голограммам. Грубый шаг необходимо выбирать с учетом периода локальных максимумов таким образом, чтобы хотя бы две точки грубого отсчета попали в интервал глобального экс- тремума. Далее на пространственный модулятор света выводятся последовательно 38 голограмм, что позволяет получить на выходе грубой оценки 37 интервалов и значения целевой функции на их границах. Интервал, ограниченный двумя наибольшими значениями функции, далее становится исходным для точной оценки величины аберрации.

Для точного вычисления амплитуды фазового искажения можно применить один из известных методов локальной оптимизации, так как на данном интервале функция будет являться унимодальной. После получения значения измеряемой аберрации алгоритм переходит либо к определению следующей аберрации, либо, если определены значения всех аберраций, завершает работу.

\section{Описание экспериментальной установки}

На рис. 5 показана оптическая схема экспериментальной установки, которая позволяет реализовать автоматическое корреляционное детектирование искажений волнового фронта, используя компьютерносинтезированные голограммы, выводимые на фазовый пространственный модулятор света отражательного ти-

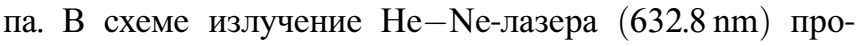
ходит через микрообъектив и точечную диафрагму и попадает на коллимирующую линзу L1 $\left(f_{1}=75 \mathrm{~mm}\right)$, установленную на моторизированную платформу для линейного перемещения Standa 8MT173-20-50. Далее коллимированный пучок, проходя через светоделительную пластину BS, попадает на фазовый модулятор света HOLOEYE Pluto $1920 \times 1080$ (шаг пикселей $8 \mu \mathrm{m}$ ). Затем пучок падает на фурье-преобразующую линзу L2 $\left(f_{2}=1000 \mathrm{~mm}\right)$, формирующую 0 и \pm 1 -й дифракционные порядки. КМОП-камера Thorlabs CS2100M-USB $(1920 \times 1080)$ установлена так, что в плоскость изображения попадает только +1-й дифракционный порядок. ПМС и камера подключены к одному компьютеру, который на основе разработанного алгоритма осуществляет

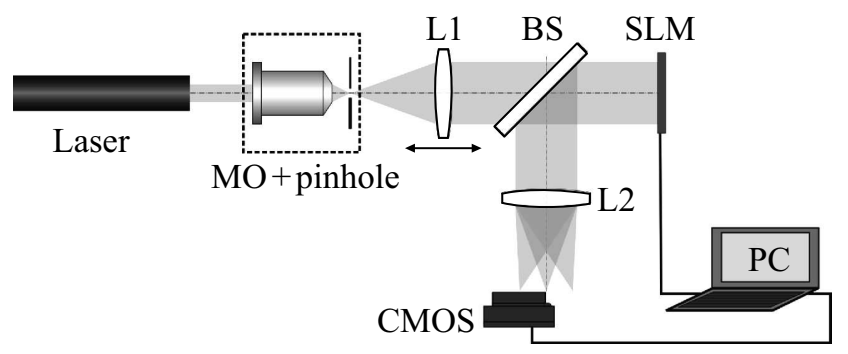

Рис. 5. Схема экспериментальной установки для автоматической оценки дефокусировки в системе; МО - микрообъектив, pinhole - точечная диафрагма, L1 - линза на моторизированной платформе, BS - делитель пучка, SLM пространственный модулятор света, L2 - фурье-объектив, CMOS - КМОП-камера. 
оценку интенсивности точки, формируемой $+1-$ д дифракционным порядком, и корректирует амплитуду полинома Цернике, кодируемого в КСГ для получения максимальной интенсивности точки.

Для контрольных измерений была выбрана аберрация дефокусировки, так как ее весьма просто можно ввести в схему физически - перемещением какого-либо оптического элемента вдоль оптической оси. В данном исследовании дефокусировка вводилась перемещением линзы L1. Теоретически в [18] было показано, что динамический диапазон данного голографического датчика может превышать $2 \lambda$. Принимая во внимания ограничения по максимальной подвижке моторизованной платформы и по дискретности шага платформы, в данном исследовании удалось задать значение дефокусировки,

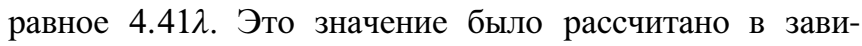
симости от смещения линзы вдоль оптической оси на основе математической интерпретации дефокусировки, описанной в [12]. Также для проверки детектирования установленной величины аберрации мы провели предварительный эксперимент: были синтезированы голо-

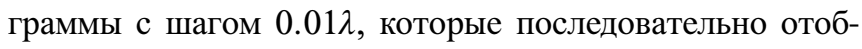
ражались на ПМС. Результат регистрации амплитуды корреляционного пика в плоскости камеры для этого эксперимента показан на рис. 6. Таким образом, истинное значение аберрации было верифицировано путем простого перебора.

После предварительного эксперимента на ПК был запущен предлагаемый алгоритм. Для грубой оценки было синтезировано 38 голограмм, амплитуда аберрации в которых менялась с шагом $0.4 \lambda$ в диапазоне $(0-15) \lambda$. Голограммы выводились на пространственный модулятор света последовательно. Частота смены кадров модулятора $60 \mathrm{~Hz}$. Массив изображения корреля-

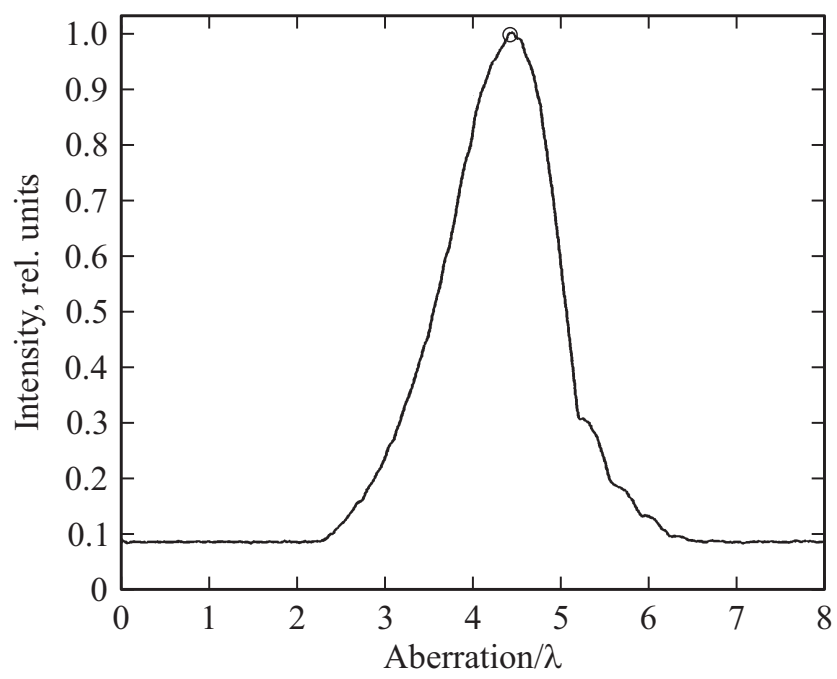

Рис. 6. График нормированной целевой функции для аберрации дефокусировки с амплитудой $4.41 \lambda$, полученный пу-

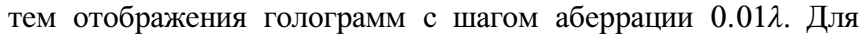
уменьшения влияния шумов камеры функция была сглажена с помощью локальной регрессии.
Измеренные значения аберраций и полученная погрешность при различном числе усредняемых кадров

\begin{tabular}{|c|c|c|c|}
\hline $\begin{array}{c}\text { Количество } \\
\text { усредняемых } \\
\text { кадров }\end{array}$ & $\begin{array}{c}\text { Введенное } \\
\text { значение }\end{array}$ & $\begin{array}{c}\text { Измеренное } \\
\text { значение }\end{array}$ & $\begin{array}{l}\text { Абсолютная } \\
\text { погрешность }\end{array}$ \\
\hline 1 & \multirow{3}{*}{$4.41 \lambda$} & $4.43 \lambda$ & $\lambda / 50$ \\
\hline 5 & & $4.419 \lambda$ & $\lambda / 110$ \\
\hline 10 & & $4.416 \lambda$ & $\lambda / 160$ \\
\hline
\end{tabular}

ционного отклика с КМОП-камеры подавался на вход вычислительного цикла, который оценивал максимум корреляционной функции для каждой конкретной голограммы. После отображения всех голограмм из массива выбирался максимум и следующая за ним по амплитуде точка. Эти точки образовывали интервал для локальной оптимизации и точного вычисления аберрации.

Для точной оценки величины аберрации на полученном интервале был использован метод НелдераМида [25]. Данный метод обладает хорошей устойчивостью к шумам, а также позволяет найти экстремум за небольшое количество итераций.

\section{Обсуждение результатов}

В таблице представлены результаты экспериментов: измеренные значения аберраций и полученная погрешность в зависимости от числа усредняемых кадров с камеры.

По таблице можно видеть, что показатели точности находятся на уровне интерферометрических аналогов и некоторых моделей датчиков Шака-Гартмана. Стоит отметить весьма значительное влияние шумов регистрирующей системы, что не позволяло проводить измерения с желаемой точностью при усреднении по одному кадру. Так, при сигнале на уровне 250-255 градации серого шум составлял 15-20 уровней серого, что эквивалентно 10\% погрешности. Этот факт можно наблюдать и на рис. 6. В нашем эксперименте без усреднения удалось достичь определения дефокусировки с точностью лишь $\lambda / 50$. Однако данное ограничение носит фундаментальный характер и при отсутствии того или иного вида фильтрации будет ограничивать точность любого метода, основывающегося на оценке амплитуды корреляционного отклика той или иной картины в плоскости регистрации.

Впрочем, если быстродействие не является ключевым параметром для задачи, в которой может быть использована такая схема измерений аберраций волнового фронта, то при усреднении по 5 кадрам камеры, что соответствует частоте порядка $10 \mathrm{~Hz}$, предлагаемый метод позволяет достичь абсолютной точности более $\lambda / 100$ в диапазоне до $5 \lambda$. Отметим, что данная 


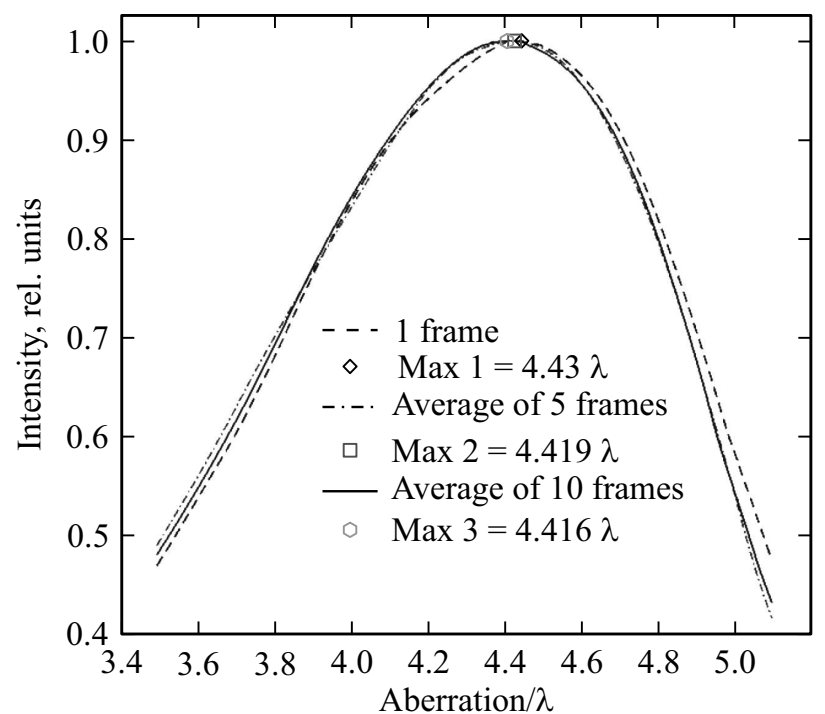

Рис. 7. График сравнения экспериментальных данных измерения аберрации дефокусировки с амплитудой $4.41 \lambda$ в различных режимах усреднения по кадрам.

точность является предельной для большинства современных интерферометрических методов измерения фазы волнового фронта, а такой диапазон измерений на ранее предложенных схемах ГДВФ реализовать было принципиально невозможно. Результаты проведения измерений аберрации дефокусировки с усреднением по нескольким кадрам представлены на рис. 7 .

Анализируя быстродействие алгоритма несколько более детально, можно утверждать, что при исключении амплитудного шума камеры существует возможность увеличить шаг грубого поиска. Так, при увеличении шага до $0.6 \lambda$ количество голограмм для отображения сократится до 25, что позволит вычислять интервал для точного поиска быстрее. Сходимость алгоритма точного поиска также существенно повысится при уменьшении влияния шумов, иными словами, количество итераций для определения одной аберрации сократится. В нашем эксперименте точное положение экстремума удавалось определить в среднем за 15 итераций, тогда как при отсутствии шума (на теоретической кривой) число итераций сокращалось вдвое.

Стоит отметить, что существует возможность применения более сложных способов оптимизации, например метода роя частиц [26], которые могут показать существенно лучшие результаты по быстродействию, не требуя при этом выполнения предварительной грубой оценки. Практическая реализация подобных алгоритмов затруднительна для рассматриваемой задачи корреляционного детектирования на основе компьютерносинтезированных голограмм и требует проведения отдельных экспериментов, однако использование столь гибких и комплексных методов глобальной оптимизации является весьма перспективным направлением исследований.

\section{Заключение}

В настоящей работе был предложен алгоритм автоматического вычисления волновых аберраций лазерного пучка в схеме голографического датчика волнового фронта. Была предложена модификация схемы, позволившая реализовать такой алгоритм на практике. Экспериментально показано, что вычисление аберраций можно проводить в два этапа: на первом этапе осуществляется грубая оценка интервала, содержащего искомое значение аберрации, а на втором этапе происходит точная оценка значения аберрации на основе методов локальной оптимизации целевой функции. Данный подход проверен при измерении дефокусировки, механически вводимой в схему путем перемещения линзы на моторизированной платформе. Показано, что предлагаемый голографический метод измерений аберраций волнового фронта позволяет получить высокую точность $\lambda / 50-\lambda / 160$ в зависимости от числа усредняемых кадров камеры. Также экспериментально подтверждена работоспособность данного метода в диапазоне измерений $5 \lambda$.

Апробация метода на большем количестве волновых аберраций, характеризующихся полиномами Цернике, осложняется необходимостью введения в схему отдельного устройства, способного имитировать различные фазовые искажения. Таким устройством может быть адаптивное зеркало или фазовый модулятор с глубиной модуляции, превышающей $2 \pi$. Однако отсутствие пространственного мультиплексирования голограмм в предложенной схеме позволяет предположить, что влияние кросс-модуляционных шумов при измерении мод Цернике высшего порядка будет существенно ниже, чем у существующих голографических датчиков.

\section{Благодарности}

Авторы благодарны В.Ю. Венедиктову и Г.Н. Вишнякову за плодотворное обсуждение задачи.

\section{Конфликт интересов}

Авторы заявляют, что у них нет конфликта интересов.

\section{Список литературы}

[1] Freisem L., Jansen G.S.M., Rudolf D., Eikema K.S.E., Witte S. // Opt. Express. 2018. V. 26. N 6. P. 6860. doi 10.1364/OE.26.006860

[2] Zhigang Jia, Kai Xu, Fengzhou Fang // Opt. Express. 2017. V. 25. N 18. P. 22125. doi 10.1364/OE.25.022125

[3] Boer Zhu, Xiangzhao Wang, Sikun Li, Guanyong Yan, Lina Shen, Lifeng Duan // Appl. Opt. 2016. V. 55. N 12. P. 3192. doi 10.1364/AO.55.003192

[4] Azucena O., Crest J., Jian Cao, Sullivan W., Kner P., Gavel D., Dillon D., Olivier S., Kubby J. // Opt. Express. 2010. V. 18. 16 P. 17521. doi 10.1364/OE.18.017521 
[5] de Visser C.C., Verhaegen M. // J. Opt. Soc. Am. A. 2013. V. 30. N 1. P. 82. doi 10.1364/JOSAA.30.000082

[6] Neil M.A.A., Booth M.J., Wilson T. // J. Opt. Soc. Am. A. 2000. V. 17. N 6. P. 1098. doi 10.1364/JOSAA.17.001098

[7] Ghebremichael F., Andersen G., Gurley K. // Appl. Opt. 2008. V. 47. P. A62. doi 10.1364/AO.47.000A62

[8] Andersen G., Dussan L., Ghebremichael F., Chen K. // Opt. Eng. 2009. V. 48. P. 085801. doi 10.1364/AO.49.005117

[9] Liu Changhai, Xi Fengjie, Ma Haotong, Huang Shengyang, Jiang Zongfu // Appl. Opt. 2010. V. 49. P. 5117. doi 10.1364/AO.49.005117

[10] Shihao Dong, Tobias Haist, Osten W. // Appl. Opt. 2012. V. 51. P. 6268. doi 10.1364/AO.51.001318

[11] Zepp A., Gtadysz S., Stein K. // Adv. Opt. Techn. 2013. V. 2. N 5-6. P. 433. doi 10.1515/aot-2013-0050

[12] Anzuola E., Zepp A., Gladysz S., Stein K. // San Diego. Proc. SPIE. 2016. V. 9979. P. 99790X. doi 10.1117/12.2236341

[13] Venediktov V.Yu. // Photonics. 2016. V. 55. N 1. P. 132.

[14] Krasin G.K., Lushnikov D.S., Odinokov S.B., Solomashenko A.B., Venediktov V.Yu., Zlokazov E.Yu. // Berlin. Proc. SPIE. 2018. V. 10787. P. 107870D. doi 10.1117/12.2325437.

[15] Orlov V.V., Venediktov V.Yu., Gorelaya A.V., Shubenkova E.V., Zhamalatdinov D.Z. // Optics \& Laser Technology. 2019. V. 116, P. 214. doi 10.1016/j.optlastec.2019.03.028

[16] Shihao Dong, Haist T., Osten W., Ruppel T., Sawodny O. // Appl. Opt. 2012. V. 51. N 9. P. 1318. doi 10.1364/AO.51.001318

[17] Konwar S., Boruah B.R. // J. Opt. Soc. Am. A. 2019. V. 36. N 5. P. 741. doi 10.1364/JOSAA.36.000741

[18] Bobrinev V.I., Galkin M.L., Kovalev M.S., Malinina P.I., Odinokov S.B. // Optoelectronics, Instrumentation Data Process. 2018. V. 54. N 1. P. 26. doi 10.3103/S8756699018010053

[19] Kovalev M.S., Krasin G.K., Odinokov S.B., Solomashenko A.B., Zlokazov E.Yu. // Opt. Express. 2019. V. 27. N 2. P. 1563. doi 10.1364/OE.27.001563

[20] Kovalev M.S., Krasin G.K., Nosov P.A., Odinokov S.B., Filippov I.Yu. // IJAER. 2017. V. 12. N 23. P. 13303.

[21] Colton D., Kress R. Inverse acoustic and electromagnetic scattering theory. N. Y.: Springer-Verlag. 2013. 406 p.

[22] Kumar B., Mahalanobis A., Juday R. Correlation Pattern Recognition. N. Y.: Cambridge University Press. 2015. 404 p.

[23] Fletcher R. Practical methods of optimization. N. Y.: WileyInterscience. 2013. 456 p.

[24] Barzilai J., Borwein J.M. // IMA Journal of Numerical Analysis. 1988. V. 8. N 1. P. 141. doi 10.1093/imanum/8.1.141.

[25] Nelder J.A., Mead R.A. // The Computer Journal. 1965. V. 7. N 4. P. 308. doi 10.1093/comjnl/7.4.308.

[26] Kennedy J., Eberhart R. // Proc. of IEEE International Conference on Neural Networks IV, Perth. 1995. P. 1942. doi 10.1109/ICNN.1995.488968 